\title{
Beneficial effects of soluble dietary Jerusalem artichoke (Helianthus tuberosus) in the prevention of the onset of type 2 diabetes and non-alcoholic fatty liver disease in high-fructose diet-fed rats
}

\author{
Wan-Ching Chang ${ }^{1} \dagger$, Huijuan $\mathrm{Jia}^{2} \dagger$, Wanping $\mathrm{Aw}^{2,3}$, Kenji Saito ${ }^{2}$, Sumio Hasegawa ${ }^{4}$ and \\ Hisanori Kato ${ }^{1,2 *}$ \\ ${ }^{1}$ Department of Applied Biological Chemistry, Graduate School of Agricultural and Life Sciences, The University of Tokyo, \\ Tokyo, Japan \\ ${ }^{2}$ Corporate Sponsored Research Program "Food for Life", Organization for Interdisciplinary Research Projects, \\ The University of Tokyo, 1-1-1, Yayoi, Bunkyo-ku, Tokyo 113-8657, Japan \\ ${ }^{3}$ Graduate School of Biomedical Science, Tokyo Medical and Dental University, Tokyo, Japan \\ ${ }^{4}$ Fuji Foods, Inc., Funabashi-shi, Chiba, Japan \\ (Submitted 18 December 2013 - Final revision received 7 May 2014 - Accepted 9 May 2014 - First published online 26 June 2014)
}

\section{Abstract}

Jerusalem artichoke (JA) has the potential to attenuate lipid disturbances and insulin resistance (IR), but the underlying mechanisms are not well understood. In the present study, we elucidated the physiological responses and mechanisms of JA intervention with a comprehensive transcriptome analysis. Wistar rats were fed a control diet, a $60 \%$ fructose-enriched diet (FRU), or a FRU with $10 \% \mathrm{JA}$ ( $n$ 6-7) for 4 weeks. An oral glucose tolerance test was carried out on day 21. Liver samples were collected for biochemical and global gene expression analyses (GeneChip ${ }^{\circledR}$ Rat Genome 2302.0 Array, Affymetrix). Fructose feeding resulted in IR and hepatic TAG accumulation; dietary JA supplementation significantly improved these changes. Transcriptomic profiling revealed that the expression of malic enzyme 1 (Me1), associated with fatty acid synthesis; decorin (Dcn), related to fibrosis; and cytochrome P450, family 1, subfamily a, polypeptide 2 (Cyp1a2) and nicotinamide phosphoribosyltransferase (Nampt), associated with inflammation, was differentially altered by the FRU, whereas dietary JA supplementation significantly improved the expression of these genes. We established for the first time the molecular mechanisms driving the beneficial effects of JA in the prevention of type 2 diabetes and non-alcoholic fatty liver disease. We propose that $10 \%$ JA supplementation may be beneficial for the prevention of the onset of these diseases.

Key words: DNA microarrays: Jerusalem artichoke: Non-alcoholic fatty liver disease: Type 2 diabetes

Over the past few decades, lifestyles have become increasingly sedentary and dietary patterns have changed, leading to an increased prevalence of obesity and diabetes ${ }^{(1)}$. According to the WHO, in 2012 at least 346 million people worldwide were suffering from diabetes, and the number of patients is increasing rapidly, especially in developing countries. Diabetes and its complications undoubtedly have a significant economic impact on individuals, families, health systems and countries. Epidemiological studies such as the Third National Health and Nutritional Examination Survey and the Dallas Heart Study have also reported the prevalence of obesity and type 2 diabetes (T2D) to be associated with non-alcoholic fatty liver disease (NAFLD). NAFLD is a spectrum of liver diseases that involves simple fat infiltration into hepatocytes (steatosis), fatty infiltration plus inflammation-non-alcoholic steatohepatitis (NASH), fibrosis and eventually cirrhosis. With the increase in the prevalence of obesity and diabetes, NAFLD has become the leading cause of chronic liver disease in developed countries ${ }^{(2)}$.

The dramatic increase in the prevalence of $\mathrm{T} 2 \mathrm{D}$ and NAFLD strongly indicates a role for environmental factors in disease pathogenesis. A vital but not well-appreciated

Abbreviations: ACC, acetyl-CoA carboxylase; CON, control; CTGF, connective tissue growth factor; CYP1A2, cytochrome P450, family 1, subfamily a, polypeptide 2; DCN, decorin; ECM, extracellular matrix; ELOVL6, elongation of very long chain fatty acids 6; FAS, fatty acid synthase; FJA, 60\% fructose-enriched diet $+10 \%$ Jerusalem artichoke powder; FRU, 60\% fructose-enriched diet; IR, insulin resistance; JA, Jerusalem artichoke; ME1, malic enzyme 1; NAFLD, non-alcoholic fatty liver disease; NAMPT, nicotinamide phosphoribosyltransferase; NASH, non-alcoholic steatohepatitis; T2D, type 2 diabetes.

*Corresponding author: Professor H. Kato, fax +8135841 1607, email akatoq@mail.ecc.u-tokyo.ac.jp

† These authors contributed equally to this study. 
change in dietary habits has been the considerable rise in the consumption of dietary fructose from sucrose and highfructose maize syrup, a common inexpensive and intense sweetener used in the food industry ${ }^{(3-5)}$. The global increase in fructose consumption is paralleled by the increase in the prevalence of obesity worldwide ${ }^{(5)}$. Fructose is rapidly metabolised by fructokinase in the liver ${ }^{(4)}$, yielding glycerol and acyl portions. The excess fructose may lead to extra TAG synthesis, thereby interrupting hepatic lipid metabolism ${ }^{(3)}$ and accelerating the development of NAFLD ${ }^{(6)}$, which may slowly progress to $\mathrm{T}_{2} \mathrm{D}^{(7)}$.

The American Diabetes Association has proposed medical nutrition therapy, recommending the intake of high doses of soluble fibre as an initial step towards the prevention of prediabetes and the management of $\mathrm{T} 2 \mathrm{D}^{(8)}$. Jerusalem artichoke (JA), a widely used root vegetable, stores carbohydrates as soluble fibres, fructans, which are polymers mainly composed of fructose units linked by $\beta$-(2-1) bonds. These linkages protect fructans from being digested like a typical fructose unit, and they are responsible for its reduced energetic value $(6.3 \mathrm{~kJ} / \mathrm{g})$ compared with typical carbohydrates $(16 \cdot 8 \mathrm{~kJ} / \mathrm{g})$, thereby contributing to the beneficial effects of dietary soluble fibre ${ }^{(9)}$. Fructans are mixtures of molecules including oligofructose and inulin. Oligofructose is defined as a fructose oligosaccharide containing two to ten monosaccharide residues, and inulin has a fructan chain length ranging from two to sixty units. A high-performance fructan has been described to contain an average degree of polymerisation of 25 . JA stores carbohydrates as high-performance soluble-type fructans (two to sixty monosaccharide residues) instead of insoluble fibres. Although the potential of fructans from JA to replace carbohydrates or fat in the management of T2D has been known since the early twentieth century ${ }^{(9)}$, the underlying properties involved have not been well defined.

The present study was conducted to examine the effects of $10 \%$ dietary JA supplementation on the physiological responses in rats fed a $60 \%$ fructose-enriched diet and to extensively elucidate the mechanisms underlying the beneficial effects of this JA intervention via a comprehensive transcriptome analysis.

\section{Materials and methods}

\section{Animals}

Male Wistar rats aged 6 weeks were obtained from Charles River (Tokyo, Japan) and housed in individual cages under controlled temperature $\left(23 \pm 2^{\circ} \mathrm{C}\right)$, humidity $(50-60 \%)$ and lighting ( $12 \mathrm{~h}$ light $-12 \mathrm{~h}$ dark cycle) conditions. Rats had ad libitum access to food and tap water. All rats were fed the MF diet (mouse feeding diet) (Oriental Yeast Company Limited), a commonly used basal diet, during a $3 \mathrm{~d}$ acclimatisation period. They were then divided into three groups (6-7 rats per group) with equal mean body weights, and they were fed the experimental diets for $28 \mathrm{~d}$. An oral glucose tolerance test was carried out on day 21, and metabolic cages were used to measure water intake and urine volume from day 24 to day 26. The animals were killed on day 28.
The present study was approved by the Animal Care and Use Committee of The University of Tokyo (approval no. P019-374), and the animals were treated in accordance with the committee's guidelines.

\section{Diets}

A mixture of dry JA root extract powder containing $52 \cdot 8 \%$ fructans was supplied by Fuji Foods, Inc. The composition of ingredients and fructans analysed by Japan Food Research Laboratories (Tokyo) is as follows: water, $4.0 \%$; protein, $13.1 \%$; fat, $1.3 \%$; ash, $5.8 \%$; carbohydrates, $75.8 \%$, of which $52 \cdot 8 \%$ are fructans (total energy per $100 \mathrm{~g}$ of JA: $1376 \cdot 8 \mathrm{~kJ}$ ). The diets used in the present experiment were based on the AIN-93G diet, and their compositions are as follows: (1) AIN-93G (control; CON); (2) 60\% fructose-enriched diet (FRU); (3) FRU + 10\% JA powder (FJA). All the three diets were adjusted with maize starch to maintain the energy balance, as shown in Table S1 (available online).

\section{Oral glucose tolerance test}

After a $16 \mathrm{~h}$ fast, $1 \mathrm{ml}$ of glucose solution $(20 \%$ in $0.9 \% \mathrm{NaCl}$ ) per $100 \mathrm{~g}$ body weight was orally administered to the rats. Blood samples were collected from the tail vein into heparinised tubes at 0, 15, 30, 60 and 120 min after glucose administration. Plasma was obtained after centrifugation (3000 rpm for $15 \mathrm{~min}$ at $4^{\circ} \mathrm{C}$ ) and stored at $-80^{\circ} \mathrm{C}$ before biochemical assays. Plasma glucose concentrations were determined using an enzyme assay (no. 439-90 901, Wako Pure Chemical Industries Limited) and insulin concentrations were measured using an ELISA kit (no. MS-301; Morinaga Institute of Biological Science, Inc.) according to the manufacturer's instructions. The AUC were calculated for glucose $\left(\mathrm{AUC}_{\text {gluc }}\right)$ and insulin $\left(\mathrm{AUC}_{\mathrm{insu}}\right)$.

\section{Biochemical assays}

After a $3 \mathrm{~h}$ fast, rats were weighed and anaesthetised intraperitoneally with pentobarbital sodium $(50 \mathrm{mg} / \mathrm{kg}$ body weight) and then killed. Blood samples were collected from the carotid artery into heparinised tubes. Plasma glucose and insulin concentrations were measured as described above. Liver samples were homogenised and lipids were extracted with chloroform-methanol $(2: 1, \mathrm{v} / \mathrm{v})$ according to the Folch method $^{(10)}$. Hepatic and plasma TAG concentrations were then measured using kits (Wako Pure Chemical Industries Limited) according to the manufacturer's instructions.

\section{Liver histological assay}

A fraction of the liver samples were embedded in Optimal Cutting Temperature Compound (Sakura Finetek) and then snap-frozen in liquid $\mathrm{N}_{2}$ and stored until further histological analysis. The tissue samples were cut into $5 \mu \mathrm{m}$ sections and stained with Oil Red O. Light microscopic detection (200X) of liver sections was performed using the Olympus BX51 System Microscope (Olympus Optical Company Limited). The percentage of lipid droplets was determined by quantification 
of the area of lipid droplets using the Threshold colour tool in ImageJ software (National Institutes of Health).

\section{Fatty acid synthase activity assay}

Total protein was extracted from $0 \cdot 1 \mathrm{~g}$ of liver tissue with $500 \mu \mathrm{l}$ of buffer (0.25 $\mathrm{M}$-sucrose, $1 \mathrm{M}$-EDTA and $3 \mathrm{M}$-Tris- $\mathrm{HCl}$ buffer; $\mathrm{pH} 7 \cdot 2$ ). The activity of fatty acid synthase (FAS) was measured in the supernatants of samples after centrifugation ( $9000 \mathrm{~g}$ for $10 \mathrm{~min}$ at $4^{\circ} \mathrm{C}$ ) using a clinical biochemical instrument, BiOLiS 24i (Tokyo Boeki Machinery Limited). The concentration of protein was measured using an N-ASSAY LTP kit (Nittobo) according to the manufacturer's instructions, and it was used to normalise the activity of FAS.

\section{Liver DNA microarray preparation, normalisation and data analysis}

Total RNA extraction and quality assessment were performed as described previously ${ }^{(11)}$. Total liver RNA from each respective group were pooled ( $n$ 6-7). Microarray analysis (GeneChip ${ }^{\circledR}$ Rat Genome 2302.0 Array, Affymetrix) was carried out as described by Jia et al. ${ }^{(11)}$. After normalisation of the differences in microarray intensities, pairwise comparisons between the CON (as a baseline) and FRU groups as well as between the FRU (as a baseline) and FJA groups were made. Differentially expressed genes were regarded as up-regulated if the fold change was greater than 1.2 and as down-regulated if it was less than $0 \cdot 8$. These genes were chosen for further analysis using the Ingenuity Pathways Analysis (Ingenuity Systems).

\section{Quantitative RT-PCR}

RT-PCR was carried as described previously ${ }^{(11)}$ to detect the expression of genes related to fatty acid synthesis, inflammation and fibrosis. Primers were designed with a web application (Primer3), and their sequences are given in Table S2 (available online). The relative amounts of mRNA were normalised to $\beta$-actin expression levels in each individual sample, and the data are presented as the fold change of normalised mRNA amounts of each sample compared with those of the CON group.

\section{Statistical analysis}

All values are presented as means with their standard errors ( $n$ 6-7). Data were analysed using one-way ANOVA, and significant differences in means were evaluated using Dunnett's test at the $P<0.05$ level.

\section{Results}

\section{General observations}

No significant difference was observed in the body weight or food intake of rats. Water intake was significantly higher $(P=0.046)$ and urine volume was higher $(P=0.084)$ in the FRU group than in the CON group, whereas significant decreases were observed in both water intake $(P=0.015)$ and urine volume $(P=0.003)$ in the FJA group. Moreover, the FJA group exhibited a significantly higher caecum weight $(P=0.000 v$. CON; $P=0.000 v$. FRU) and lower $\mathrm{pH}$ levels of caecal contents $(P=0.001 \quad v$. CON; $P=0.000$ $v$. FRU) compared with the other groups. Plasma TAG concentrations were lower $(P=0 \cdot 140)$ and hepatic TAG concentrations were significantly decreased $(P=0.006)$ in the FJA group compared with those in the FRU group (Table 1).

\section{Oral glucose tolerance test}

At $30 \mathrm{~min}$ after oral glucose administration, there was an increase $(P=0.064)$ in the plasma glucose concentrations of FRU group when compared with those of the CON group, and a significant decrease $(P=0.001)$ was observed in the FJA group when compared with the concentrations of the FRU group (Fig. 1(A) and (B)). In addition, the FRU group exhibited a significantly higher insulin peak compared with

Table 1. General characteristics of the experimental groups (Mean values with their standard errors)

\begin{tabular}{|c|c|c|c|c|c|c|}
\hline & \multicolumn{2}{|c|}{ CON } & \multicolumn{2}{|c|}{ FRU } & \multicolumn{2}{|c|}{ FJA } \\
\hline & Mean & SE & Mean & SE & Mean & SE \\
\hline Body weight (g) & 393.5 & $7 \cdot 2$ & 393.9 & $16 \cdot 1$ & $377 \cdot 2$ & 11.0 \\
\hline Total food intake $(\mathrm{g})$ & 661.5 & 11.6 & 676.6 & 28.5 & $601 \cdot 8$ & $27 \cdot 8$ \\
\hline $3 \mathrm{~d}$ water intake $(\mathrm{ml})$ & $105 \cdot 3^{a}$ & $7 \cdot 0$ & $127 \cdot 3^{\mathrm{b}}$ & 9.2 & $99 \cdot 7^{a}$ & $6 \cdot 3$ \\
\hline $3 \mathrm{~d}$ urine volume $(\mathrm{ml})$ & $49 \cdot 9^{\mathrm{a}, \mathrm{b}}$ & $6 \cdot 6$ & $62 \cdot 3^{\mathrm{a}}$ & $5 \cdot 3$ & $38.9^{\mathrm{b}}$ & 4.8 \\
\hline Caecal content $(\mathrm{g})$ & $0.9^{a}$ & 0.1 & $0.9^{a}$ & 0.0 & $1.4^{\mathrm{b}}$ & 0.0 \\
\hline Caecal pH & $8 \cdot 3^{\mathrm{a}}$ & 0.1 & $8 \cdot 3^{a}$ & 0.1 & $7 \cdot 1^{\mathrm{b}}$ & 0.3 \\
\hline Glucose $(\mathrm{mmol} / \mathrm{l})$ & $8 \cdot 7^{\mathrm{a}}$ & 0.4 & $10 \cdot 5^{b}$ & 0.5 & $10 \cdot 1^{a, b}$ & 0.4 \\
\hline Insulin (pmol/l) & $766 \cdot 0$ & $86 \cdot 2$ & $1059 \cdot 2$ & $162 \cdot 2$ & 813.9 & $96 \cdot 3$ \\
\hline Plasma TAG (mmol/l) & 1.8 & 0.2 & $2 \cdot 8$ & 0.4 & $2 \cdot 3$ & 0.3 \\
\hline Liver weight (g) & 14.6 & 0.3 & $16 \cdot 0$ & 0.8 & $16 \cdot 0$ & 0.8 \\
\hline Hepatic TAG $(\mu \mathrm{mol} / \mathrm{g}$ liver $)$ & $15 \cdot 9^{a, b}$ & 1.5 & $17 \cdot 8^{\mathrm{a}}$ & $2 \cdot 4$ & $10 \cdot 3^{\mathrm{b}}$ & 0.9 \\
\hline
\end{tabular}


(A)

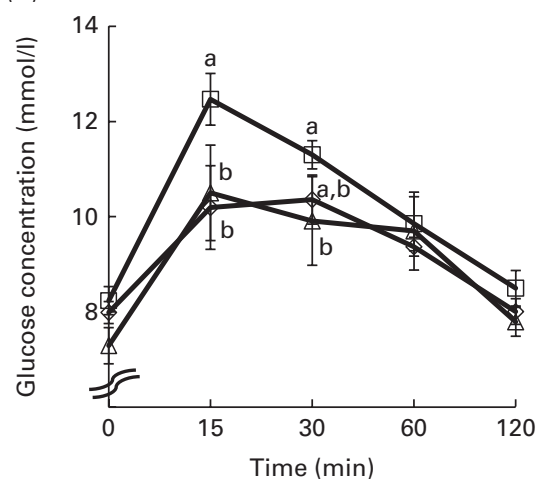

(C)

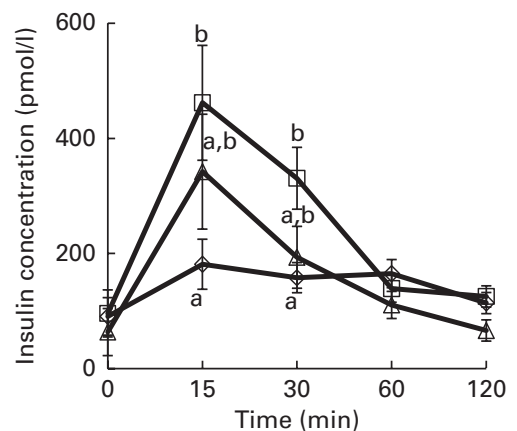

(B)

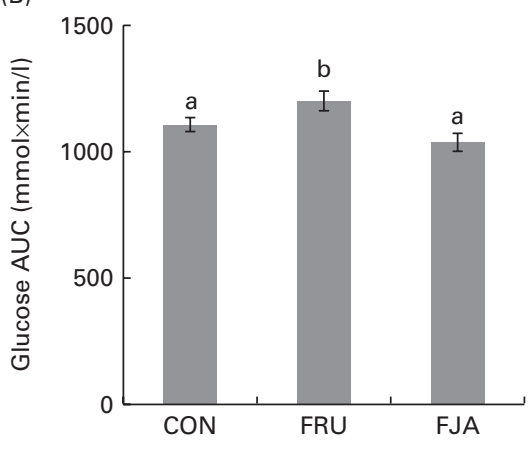

(D)

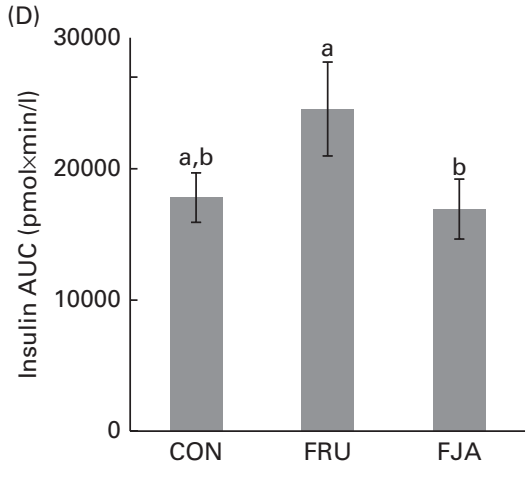

Fig. 1. Glucose and insulin concentrations during the oral glucose tolerance test (OGTT). The OGTT was carried out after the rats had been fasted for $16 \mathrm{~h}$ on day 21. (A) Plasma glucose concentration and (B) area under the glucose curve. (C) Plasma insulin concentration and (D) area under the insulin curve in response to glucose administration at different time points. Values are means $(n 6-7)$, with their standard errors represented by vertical bars. ${ }^{a, b}$ Mean values with unlike letters were significantly different $(P<0.05$; Dunnett's test). CON $(\diamond)$, Control; FRU $(\square), 60 \%$ fructose-enriched diet; FJA $(\Delta), 60 \%$ fructose-enriched diet with $10 \%$ Jerusalem artichoke supplementation.

the CON group at $15 \mathrm{~min}(P=0 \cdot 017)$ and $30 \mathrm{~min}(P=0 \cdot 010$ Fig. $1(\mathrm{C})$ and (D)). $\mathrm{AUC}_{\text {gluc }}(P=0.006)$ and $\mathrm{AUC}_{\text {insu }}$ $(P=0.049)$ in the FJA group were significantly decreased when compared with those in the FRU group.

\section{Hepatic lipid accumulation and fatty acid synthase activity}

Accumulation of evident lipid droplets was observed in the liver of the FRU group. The $10 \%$ dietary JA supplementation decreased both the size and number of lipid droplets (Fig. 2(A)), and a significant reduction $(P=0 \cdot 011)$ was also observed after quantifying hepatic lipid depositions in the FJA group compared with those in the FRU group (Fig. 2(B)). Fructose feeding led to a significant increase $(P=0.000)$ in FAS activity, which was reduced by dietary JA supplementation, although this change was not significant $(P=0.058$; Fig. 2(C)).

\section{Differentially expressed genes in the liver}

There were 147 genes that were differentially expressed, of which seventy-five were up-regulated and seventy-two genes were down-regulated in the FJA group compared with the FRU group. These differentially expressed genes are further summarised in Table S3 (available online).

\section{Expression of genes related to fatty acid synthesis and hepatic fibrosis}

High-fructose diet feeding for 4 weeks led to the up-regulation of the expression of the following genes involved in fatty acid synthesis: elongation of very long chain fatty acids 6 (Elovl6; fold change - FJA $v$. FRU: 0.8; FRU $v$. CON: 2.1); acetyl-CoA carboxylase (AcC; FJA $v$. FRU: 0.8; FRU $v$. CON: 1.7 ); malic enzyme 1 (Me1; FJA $v$. FRU: 0.8; FRU $v$. CON: $2 \cdot 5$ ); fatty acid desaturase 2 (Fads2; FJA $v$. FRU: 0.8; FRU $v$. CON: 1.2); acetyl-CoA carboxylase- $\alpha$ (Acaca; FJA $v$. FRU: 0.8; FRU $v$. CON: 1.3). The expression of these genes was subsequently down-regulated after treatment with JA for $28 \mathrm{~d}$.

The expression of the following genes associated with NAFLD leading to cirrhosis was similarly found to be up-regulated during fructose feeding: insulin-like growth factor-binding protein 1 (Igfbp1; fold change - FJA $v$. FRU: $0 \cdot 8$; FRU $v$. CON: 1.2 ); decorin (Dcn; FJA $v$. FRU: 0.8; FRU $v$. CON: 1.2); connective tissue growth factor (Ctgf; FJA $v$. FRU: $0 \cdot 8$; FRU $v$. CON: $1 \cdot 4$ ). The expression of these genes was downregulated by dietary JA supplementation. Conversely, the expression of nicotinamide phosphoribosyltransferase (Nampt; FJA $v$. FRU: 1.4; FRU $v$. CON: 0.5) and cytochrome P450, family 1 , subfamily a, polypeptide 2 (Cyp1a2; FJA $v$. FRU: 1.4; FRU $v$. CON: 0.6) was down-regulated in the FRU group and increased by dietary JA supplementation. 
(A)

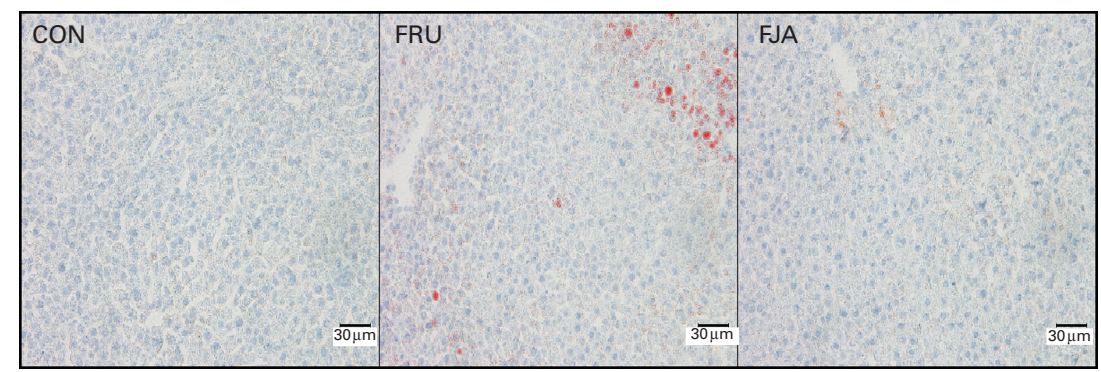

(B)

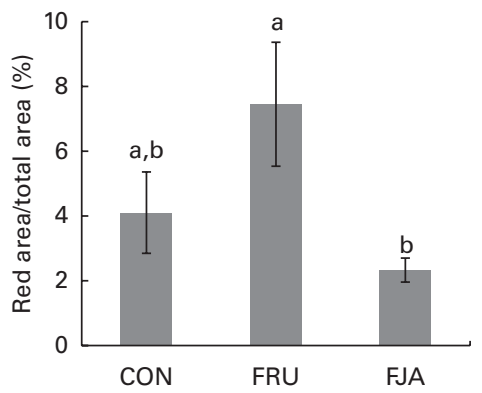

(C)

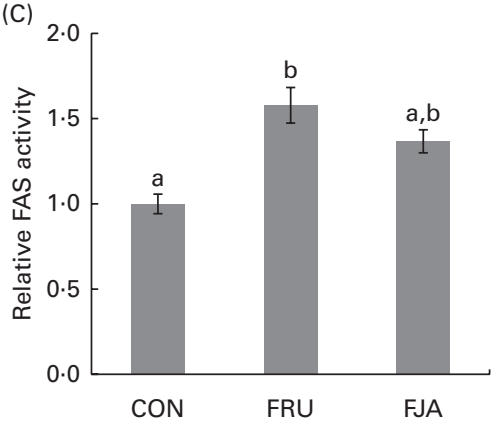

Fig. 2. Liver histology and fatty acid synthase (FAS) activity in fructose-fed rats. (A) Oil Red O staining of liver sections from rats of each treatment group. (B) Percentage of lipid droplets determined by quantification of the area of lipid droplets by the Threshold colour tool in ImageJ software. (C) FAS activity normalised by total protein. Values are means $(n 6-7)$, with their standard errors represented by vertical bars. ${ }^{a, b}$ Mean values with unlike letters were significantly different $(P<0.05$; Dunnett's test). CON, control; FRU, $60 \%$ fructose-enriched diet; FJA, $60 \%$ fructose-enriched diet with $10 \%$ Jerusalem artichoke supplementation. (A colour version of this figure can be found online at http://www.journals.cambridge.org/bjn)

\section{Validation of microarray analysis results with $R T-P C R$}

The differential expression of eleven selected genes was examined using RT-PCR (Fig. 3): Elovl6; Acc; Me1; Fas; Fads2; Acaca; Igfbp1; Dcn; Ctgf; Cyp1a2; Nampt. All the results exhibited high consistency with those of the microarray analysis. Fructose feeding induced a significant alteration in the expression of Dcn $(P=0.043)$, Cyp1a2 $(P=0.007), M e 1$ $(P=0.000)$ and Elovl6 $(P=0.010)$ compared with the expression in the CON group. The expression of $D c n$ $(P=0.016), \operatorname{Cyp1a2}(P=0.019)$ and $M e 1(P=0.003)$ was significantly ameliorated by dietary JA supplementation, whereas there was a decrease in the expression of Elovl6 $(P=0.092)$. There was also a significant mitigation $(P=0.027)$ of Nampt expression when compared with that in the FJA and FRU groups, although the increase in the expression in the FRU group compared with that in the CON group was not significant $(P=0 \cdot 129)$. The expression of the other genes, Acc, Fas, Fads2, Acaca and Igfbp1, was attenuated by dietary JA supplementation.

\section{Discussion}

High fructose intake has been reported to be associated with numerous metabolic abnormalities in humans and laboratory animals, and it does not result in a significant weight gain. In rodents, 4 weeks of high-fructose diet feeding is similar to a modern dietary lifestyle in humans ${ }^{(5)}$. In the present study, after 4 weeks of treatment, fructose feeding resulted in significantly higher plasma glucose and insulin concentrations, elevated plasma and hepatic TAG concentrations, and the development of hepatic fat droplets. In contrast, dietary JA supplementation led to an improvement in insulin resistance (IR) and significantly decreased hepatic TAG accumulation. Our comprehensive analysis of global gene expression also revealed an association between JA intervention and significant alterations in the expression of genes related to fatty acid synthesis, fibrosis and inflammation. JA may thus be potentially useful as a countermeasure against obesity, inflammation, and hepatic fibrosis and ultimately in the prevention of T2D and NAFLD.

Fructans have been reported to lower plasma TAG concentrations and prevent TAG accumulation induced by fructose in the liver ${ }^{(12)}$. The present results, consistent with this finding, showed that the consumption of a FRU led to increased plasma and hepatic TAG concentrations (Table 1), whereas dietary JA supplementation reduced plasma TAG concentrations by $18 \%$ and significantly decreased hepatic TAG concentrations ( $42 \%$ ). Liver sections stained with Oil Red O also revealed the accumulation of lipid droplets in the FRU group, but not in the FJA group (Fig. 2(A)).

As fructose is readily absorbed and rapidly metabolised by the liver, the exposure of the liver to high fructose amounts leads to a rapid stimulation of lipogenesis and TAG accumulation, which in turn contributes to reduced insulin sensitivity and increased IR and/or glucose intolerance ${ }^{(3)}$. This can be attributed to fructose-1-phosphate activating dual-specificity mitogen-activated protein kinase kinase 7 , which stimulates 
(A)

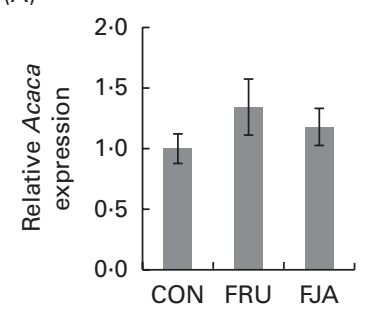

(D)

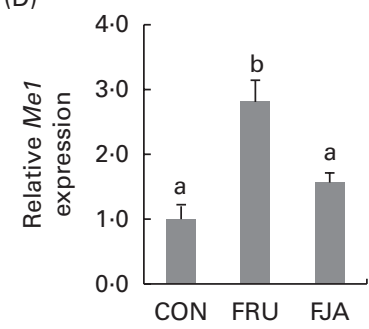

(G)

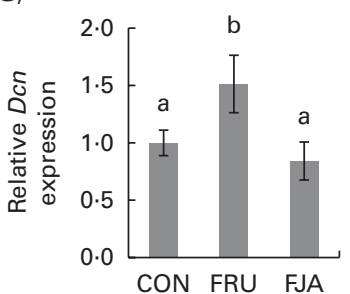

(J)

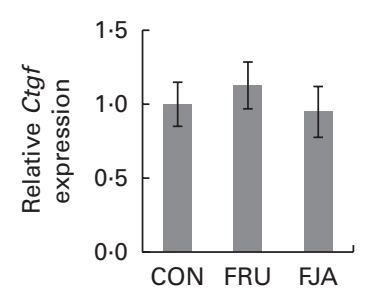

(B)

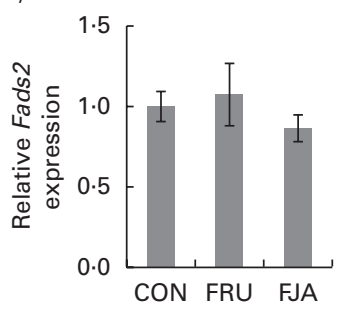

(E)

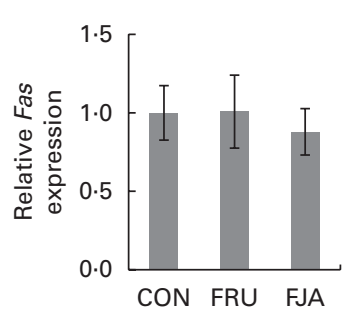

(H)
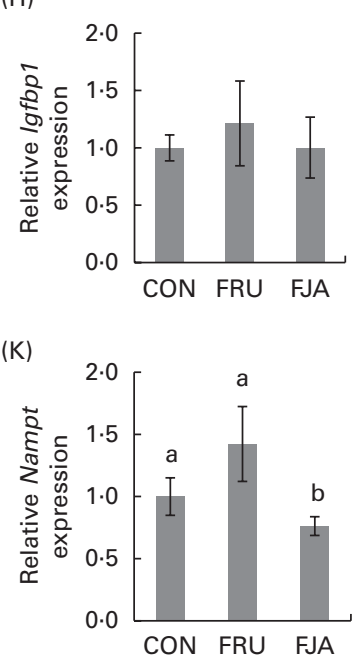

(I)

(C)

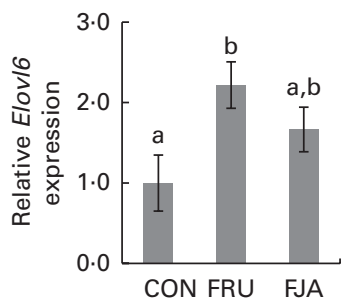

(F)
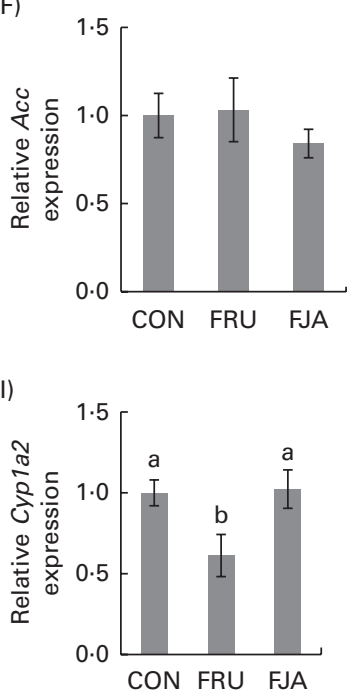

Fig. 3. Hepatic mRNA expression of genes related to fatty acid synthesis, inflammation and fibrosis. The relative mRNA expression was measured by RT-PCR. The following genes are related to fatty acid synthesis: (A) acetyl-CoA carboxylase- $\alpha$ (Acaca); (B) fatty acid desaturase 2 (Fads2); (C) elongation of very long chain fatty acids 6 (Elov/6); (D) malic enzyme 1 (Me1); (E) fatty acid synthase (Fas); (F) acetyl-CoA carboxylase (Acc). The following genes are associated with non-alcoholic fatty liver disease: (G) decorin $(D c n) ;(H)$ insulin-like growth factor-binding protein 1 (Igfbp 1); (I) cytochrome P450, family 1, subfamily a, polypeptide 2 (Cyp1a2); $(\mathrm{J})$ connective tissue growth factor (Ctgf); $(\mathrm{K})$ nicotinamide phosphoribosyltransferase (Nampt). Values are means of the fold changes normalised to $\beta$-actin mRNA expression, with their standard errors represented by vertical bars. ${ }^{\mathrm{a}, \mathrm{b}}$ Mean values with unlike letters were significantly different $(P<0.05$; Dunnett's test). CON, control; FRU, $60 \%$ fructose-enriched diet; FJA, $60 \%$ fructose-enriched diet with $10 \%$ Jerusalem artichoke supplementation.

the hepatic enzyme mitogen-activated protein kinase $8^{(13,14)}$. The intermediate diacylglycerol, which accumulates during de novo lipogenesis, activates hepatic protein kinase $\mathrm{C} \varepsilon$ type ${ }^{(15)}$. Both mitogen-activated protein kinase and protein kinase $\mathrm{C} \varepsilon$ trigger serine phosphorylation and the subsequent inactivation of insulin receptor substrate 1 , which leads to hepatic $\operatorname{IR}^{(16,17)}$.

The present results demonstrated that the FRU group had increased glucose and insulin concentrations, whereas the results of the oral glucose tolerance test indicated a significant decrease in the FJA group (Fig. 1). In addition, the fructose-fed rats also exhibited symptoms of T2D such as polydipsia and polyuria (Table 1), and dietary JA supplementation ameliorated some T2D symptoms, i.e. significantly decreased water intake and urine volume. The suppression of lipogenesis and reduction of TAG accumulation induced by JA might thus result in increased insulin sensitivity.

Based on the DNA microarray data, two of the top five canonical pathways ranked according to the Ingenuity Pathways Analysis (Ingenuity Systems) were the fatty acid synthesis and pyruvate metabolism pathways. The expression of Elovl6, Me1, Acc and Fas was up-regulated in the fructose-fed rats, but down-regulated in the FJA group. Elovl6, Me1, Acc and Fas are important genes involved in hepatic fatty acid synthesis and pyruvate metabolism pathways. ELOVL6 is a key microsomal enzyme involved in the elongation of SFA and MUFA with twelve, fourteen and sixteen carbons and has been reported to induce IR in fatty liver ${ }^{(18)}$, and its hepatic expression is positively related to $\mathrm{NASH}^{(19)}$. The expression of ELOVL6 has been reported to be elevated in the liver of 
NAFLD patients and to be positively correlated with steatosis and serum alanine aminotransferase concentrations ${ }^{(19)}$

ME1 catalyses the reversible oxidative decarboxylation of malate to pyruvate, $\mathrm{CO}_{2}$ and $\mathrm{NADPH}$, with the latter contributing to de novo fatty acid synthesis ${ }^{(20)}$. In addition, the up-regulation of Me1 expression is associated with a predisposition to $\mathrm{T}_{2} \mathrm{D}^{(21)}$. Acc encodes the biotin-dependent enzyme ACC, which catalyses the irreversible carboxylation of acetyl-CoA to produce malonyl-CoA through its two catalytic activities, biotin carboxylase and carboxyltransferase. The key role of ACC is to provide the malonyl-CoA substrate for the biosynthesis of fatty acids. Mice deficient in Acc exhibit continuous fatty acid oxidation and reduced body fat and body weight; as such, this gene is a target for drug development against obesity, diabetes and other symptoms of the metabolic syndrome ${ }^{(22)}$. Fas encodes the enzyme FAS, which is a multi-enzyme protein involved in fatty acid synthesis ${ }^{(23)}$. FAS primarily catalyses the synthesis of palmitate from acetyl-CoA and malonyl-CoA in the presence of NADPH, yielding long-chain $\mathrm{SFA}^{(23)}$. It has been reported that NAFLD patients have more amounts of FAS protein in the liver, indicating elevated fatty acid synthesis in hepatic cells ${ }^{(24)}$. FAS is also associated with insulin sensitivity, which is activated when there is high TAG production. Consistent with the above-mentioned findings, we observed that the high-fructose diet resulted in significantly increased FAS activity, whereas dietary JA supplementation decreased this activity (Fig. 2(C)). The transcriptome analysis demonstrated that dietary JA supplementation may act by down-regulating the expression of Elovl6, Me1, Acc and Fas, thereby moderating pyruvate and fatty acid metabolism, delaying hepatic lipid metabolism and thus ameliorating T2D symptoms.
In the obese state, a number of changes occur in the adipose tissue including mature adipocyte expansion, new adipocyte formation, inflammatory cell accumulation in the adipose tissue, and neovascularisation to provide blood to the expanding adipose tissue. To adapt to these changes, remodelling of the extracellular matrix (ECM) occurs by degradation of the existing ECM, and the production of new ECM is required $^{(25,26)}$. Dcn encodes the protein DCN, the expression of which is markedly up-regulated in the adipose tissue in the obese state and may therefore play a role in adipose tissue homeostasis or in the pathophysiology associated with obesity $^{(25)}$. Its hepatic expression has been reported to be up-regulated in hepatic fibrosis and cirrhosis. As DCN is required for the correct folding of other ECM components such as collagen, elevated levels of DCN may be induced in the adipose tissue in the obese state to facilitate the formation of new ECM structures within the expanding adipose tissue $^{(27)}$. DCN can also regulate collagen fibrillogenesis and modulate growth factor activity, and the levels of its mRNA expression increase in rat liver during fibrosis and cirrhosis ${ }^{(26)}$

CTGF is an intermediate key molecule involved in the pathogenesis of chronic liver diseases and its expression is up-regulated in hyperglycaemic conditions ${ }^{(28)}$. CTGF also plays vital roles in the activation of hepatic stellate cells and the progression of fibrosis ${ }^{(29)}$. The induction of CTGF by insulin in hepatic stellate cells also suggests a role for insulin in the fibrogenic process during $\mathrm{NASH}^{(30)}$. Data in the last few years have provided compelling evidence that CTGF mRNA and protein levels are correlated with the degree of hepatic fibrosis, irrespective of the type of disease ${ }^{(30)}$. In the present study, the FRU induced an up-regulation of hepatic Ctgf expression, and this increase was attenuated by the $10 \%$ dietary JA

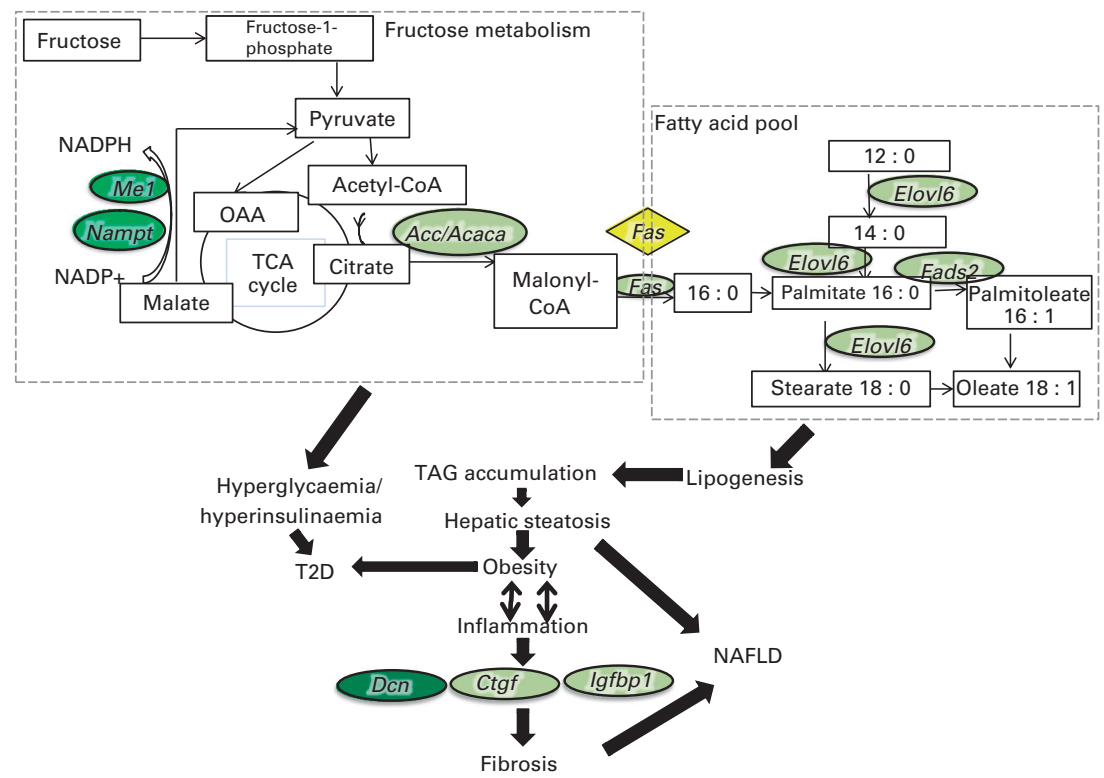

Fig. 4. Schematic representation of the metabolic changes induced by the fructose-enriched diet and $10 \%$ Jerusalem artichoke (JA) supplementation in rats. The genes in light green ovals had a strong tendency, whereas the genes in dark green ovals indicate that a significant $(P<0.05)$ improvement was caused by JA supplementation. Me1, malic enzyme 1; Acc, acetyl-CoA carboxylase; Acaca, acetyl-CoA carboxylase- $\alpha$; Fas, fatty acid synthase; Elovl6, elongation of very long chain fatty acids 6; Fads2, fatty acid desaturase 2; T2D, type 2 diabetes; Dcn, decorin; Ctgf, connective tissue growth factor; Igfbp1, insulin-like growth factor-binding protein 1; NAFLD, non-alcoholic fatty liver disease; Nampt, nicotinamide phosphoribosyltransferase; TCA, tricarboxylic acid. 
supplementation, indicating that JA may, via the suppression of Ctgf and Dcn expression, prevent the onset of fibrosis, inhibiting the progress of NAFLD.

On the other hand, a new functional link between NAD metabolism and inflammation has been established, indicating that NAD-dependent enzymes may regulate proinflammatory cytokine production. Nampt encodes the enzyme NAMPT, a rate-limiting component of the mammalian NAD biosynthesis pathway from nicotinamide ${ }^{(31)}$. In addition, NAMPT has been reported to be associated with TAG, hepatic enzymes and proinflammatory markers, indicating a role in dyslipidaemia, fatty liver disease and low-grade chronic inflammation that accompany obesity ${ }^{(32)}$. In the present study, fructose feeding was found to induce the accumulation of TAG and up-regulate the activity of FAS, which is a hepatic enzyme involved in fatty acid synthesis. In contrast, dietary JA supplementation led to a significant improvement in TAG accumulation and down-regulated FAS activity. This phenomenon may be due, in part, to the reduced expression of Nampt.

The expression of cyp1a2 is commonly found to be decreased in the presence of the proinflammatory cytokines TNF $\alpha$ and IL- ${ }^{(33)}$, during NAFLD progression and also in cirrhotic liver patients ${ }^{(34,35)}$. The expression of Cyp1a2, which is commonly found to be decreased in patients with NAFLD ${ }^{(34)}$, was similarly decreased in the FRU group and increased by dietary JA supplementation. In obese subjects, all tissues experience an increase in inflammatory exposure, including the liver ${ }^{(36)}$. Fructose feeding resulted in the down-regulation of the expression of Cyp1a2, and this alteration was significantly mitigated by JA intervention. We can infer that fructose feeding may have resulted in a decrease in the levels of proinflammatory cytokines, although the change in their expression was not significant.

NAFLD consists of a benign form of hepatic steatosis and the more perilous NASH, which may result in cirrhosis and hepatic failure. Patients with NAFLD are more susceptible to dyslipidaemia, increased IR and elevated blood pressure compared with individuals of a similar weight without NAFLD ${ }^{(37)}$, which is observed in fructose-fed rats.

In the present study, dietary JA supplementation improved hepatic TAG accumulation as well as IR, despite the absence of a change in blood pressure. NAFLD consists of a 'two-hit' hypothesis, where the first 'hit' is the development of intrahepatic lipid and hepatic steatosis, resulting in an imbalance of normal hepatic lipid metabolism, which is attributable to excessive lipid influx, impaired lipid clearance, or both. At this juncture, steatosis is potentially reversible and does not necessarily lead to permanent hepatic injury ${ }^{(38)}$. Similarly to NAFLD patients, in the present study, rats in the FRU group exhibited hepatic TAG accumulation and hepatic steatosis, which were alleviated by JA intake. The second, less common but more virulent 'hit', which occurs in 5\% of individuals with steatosis, is a concomitant inflammatory process leading to ballooning degeneration and perisinusoidal fibrosis, which promote apoptosis and hepatocellular death, resulting in scarring and progression to $\mathrm{NASH}^{(38)}$.
Fructose feeding increased the expression of the cytokine marker Nampt and genes involved in fibrosis, Dcn and Ctgf, whereas dietary JA supplementation mediated these effects, indicating that JA suppresses the inflammatory pathway, leading to the prevention of fibrosis.

JA has traditionally been known as a robust functional food that reduces glucose and TAG concentrations and cholesterol accumulation, thereby revealing its anti-obesity properties. In the present study, we validated not only the anti-obesity and anti-diabetic properties, but also the anti-fibrotic and antiinflammatory properties of JA supplementation via microarray technology, which is a powerful and commonly used tool for the global evaluation of gene expression profiles in tissues. This technique not only provides a considerable amount of information on markers and predictive factors that may potentially characterise a specific clinical picture, but also promises new applications for therapy ${ }^{(11,39)}$.

To our knowledge, this is an original study that has elucidated the molecular mechanisms underlying the beneficial effects of JA that may play a role in the prevention of T2D by down-regulating the expression of Me1 and Fas involved in fatty acid synthesis and reducing the expression of Dcn, Nampt and Cyp1a2 associated with NAFLD (Fig. 4). Based on the findings of the present study, we propose that $10 \%$ JA supplementation may be used as a potential therapeutic approach for preventing the onset of T2D as well as its hepatic manifestation, NAFLD.

\section{Supplementary material}

To view supplementary material for this article, please visit http://dx.doi.org/10.1017/S0007114514001421

\section{Acknowledgements}

The present study did not receive any financial support.

The authors' contributions are as follows: W.-C. C., H. J. contributed to the study design, data collection, manuscript writing; W. A. carried out the data analysis; K. S. carried out the statistical analysis; S. H. collected the samples; H. J., W. A., K. S. and H. K. revised the manuscript; H. K. was responsible for the supervision of the study. All the authors approved the final manuscript.

None of the authors has any conflicts of interest to declare.

\section{References}

1. de Alwis NMW \& Day CP (2008) Non-alcoholic fatty liver disease: the mist gradually clears. J Hepatol 48, S104-S112.

2. Browning JD, Szczepaniak LS, Dobbins R, et al. (2004) Prevalence of hepatic steatosis in an urban population in the United States: impact of ethnicity. Hepatology $\mathbf{4 0}$, 1387-1395.

3. Basciano H, Federico L \& Adeli K (2005) Fructose, insulin resistance, and metabolic dyslipidemia. Nutr Metab (Lond) 2,5 .

4. Hallfrisch J (1990) Metabolic effects of dietary fructose. FASEB J 4, 2652-2660. 
5. Goran MI, Ulijaszek SJ \& Ventura EE (2013) High fructose corn syrup and diabetes prevalence: a global perspective. Glob Public Health 8, 55-64.

6. Vos MB \& Lavine JE (2013) Dietary fructose in nonalcoholic fatty liver disease. Hepatology 57, 2525-2531.

7. Lv W-S, Sun RX, Gao YY, et al. (2013) Nonalcoholic fatty liver disease and microvascular complications in type 2 diabetes. World J Gastroenterol 19, 3134-3142.

8. Franz MJ, Powers MA, Leontos C, et al. (2010) The evidence for medical nutrition therapy for type 1 and type 2 diabetes in adults. J Am Diet Assoc 110, 1852-1889.

9. Niness KR (1999) Inulin and oligofructose: what are they? J Nutr 129, 1402S-1406S.

10. Folch J, Lees M \& Sloane Stanley GH (1957) A simple method for the isolation and purification of total lipides from animal tissues. J Biol Chem 226, 497-509.

11. Jia H, Saito K, Aw W, et al. (2013) Transcriptional profiling in rats and an ex vivo analysis implicate novel beneficial function of egg shell membrane in liver fibrosis. J Funct Foods 5, 1611-1619.

12. Busserolles J, Gueux E, Rock E, et al. (2003) Oligofructose protects against the hypertriglyceridemic and pro-oxidative effects of a high fructose diet in rats. J Nutr 133, 1903-1908.

13. Wei Y, Wang D, Topczewski F, et al. (2007) Fructosemediated stress signaling in the liver: implications for hepatic insulin resistance. $J$ Nutr Biochem 18, 1-9.

14. Hirosumi J, Tuncman G, Chang L, et al. (2002) A central role for JNK in obesity and insulin resistance. Nature $\mathbf{4 2 0}$, $333-336$.

15. Morino K, Petersen KF \& Shulman GI (2006) Molecular mechanisms of insulin resistance in humans and their potential links with mitochondrial dysfunction. Diabetes 55, S9-S15.

16. Bezerra RM, Ueno M, Silva MS, et al. (2000) A high fructose diet affects the early steps of insulin action in muscle and liver of rats. J Nutr 130, 1531-1535.

17. Wei Y \& Pagliassotti MJ (2004) Hepatospecific effects of fructose on c-jun NH2-terminal kinase: implications for hepatic insulin resistance. Am J Physiol Endocrinol Metab 287, E926-E933.

18. Matsuzaka T, Shimano H, Yahagi N, et al. (2007) Crucial role of a long-chain fatty acid elongase, Elovl6, in obesityinduced insulin resistance. Nat Med 13, 1193-1202.

19. Matsuzaka T, Atsumi A, Matsumori R, et al. (2012) Elovl6 promotes nonalcoholic steatohepatitis. Hepatology $\mathbf{5 6}$, 2199-2208.

20. Wise EM Jr \& Ball EG (1964) Malic enzyme and lipogenesis. Proc Natl Acad Sci US A 52, 1255-1263.

21. Zhong H, Beaulaurier J, Lum PY, et al. (2010) Liver and adipose expression associated SNPs are enriched for association to type 2 diabetes. PLoS Genet 6, e1000932.

22. Tong L (2005) Acetyl-coenzyme A carboxylase: crucial metabolic enzyme and attractive target for drug discovery. Cell Mol Life Sci 62, 1784-1803.

23. Alberts AW, Strauss AW, Hennessy S, et al. (1975) Regulation of synthesis of hepatic fatty acid synthetase: binding of fatty acid synthetase antibodies to polysomes. Proc Natl Acad Sci U S A 72, 3956-3960.

24. Yang Z-X, Shen W \& Sun H (2010) Effects of nuclear receptor FXR on the regulation of liver lipid metabolism in patients with non-alcoholic fatty liver disease. Hepatol Int $\mathbf{4}$ 741-748.

25. Bolton K, Segal D, McMillan J, et al. (2008) Decorin is a secreted protein associated with obesity and type 2 diabetes. Int J Obes (Lond) 32, 1113-1121.

26. Shi Y-F, Zhang Q, Cheung P-Y, et al. (2006) Effects of rhDecorin on TGF- $\beta 1$ induced human hepatic stellate cells LX-2 activation. Biochim Biophys Acta 1760, 1587-1595.

27. Iozzo RV \& Murdoch AD (1996) Proteoglycans of the extracellular environment: clues from the gene and protein side offer novel perspectives in molecular diversity and function. FASEB J 10, 598-614.

28. Murphy M, Godson C, Cannon S, et al. (1999) Suppression subtractive hybridization identifies high glucose levels as a stimulus for expression of connective tissue growth factor and other genes in human mesangial cells. $J$ Biol Chem 274, 5830-5834.

29. Rachfal AW \& Brigstock DR (2003) Connective tissue growth factor (CTGF/CCN2) in hepatic fibrosis. Hepatol Res 26, 1-9.

30. Paradis V, Perlemuter G, Bonvoust F, et al. (2001) High glucose and hyperinsulinemia stimulate connective tissue growth factor expression: a potential mechanism involved in progression to fibrosis in nonalcoholic steatohepatitis. Hepatology 34, 738-744.

31. Revollo JR, Grimm AA \& Imai S (2004) The NAD biosynthesis pathway mediated by nicotinamide phosphoribosyltransferase regulates Sir2 activity in mammalian cells. $J$ Biol Chem 279, 50754-50763.

32. Catalán V, Gómez-Ambrosi J, Rodríguez A, et al. (2011) Association of increased visfatin/PBEF/NAMPT circulating concentrations and gene expression levels in peripheral blood cells with lipid metabolism and fatty liver in human morbid obesity. Nutr Metab Cardiovasc Dis 21, 245-253.

33. Zhou M, Maitra SR \& Wang P (2008) The potential role of transcription factor aryl hydrocarbon receptor in downregulation of hepatic cytochrome P-450 during sepsis. Int J Mol Med 21, 423-428.

34. Congiu M, Mashford ML, Slavin JL, et al. (2002) UDP glucuronosyltransferase mRNA levels in human liver disease. Drug Metab Dispos 30, 129-134.

35. Fisher CD, Lickteig AJ, Augustine LM, et al. (2009) Hepatic cytochrome $\mathrm{P} 450$ enzyme alterations in humans with progressive stages of nonalcoholic fatty liver disease. Drug Metab Dispos 37, 2087-2094.

36. Cai D, Yuan M, Frantz DF, et al. (2005) Local and systemic insulin resistance resulting from hepatic activation of IKKbeta and NF-kappaB. Nat Med 11, 183-190.

37. Deivanayagam S, Mohammed BS, Vitola BE, et al. (2008) Nonalcoholic fatty liver disease is associated with hepatic and skeletal muscle insulin resistance in overweight adolescents. Am J Clin Nutr 88, 257-262.

38. Paschos P \& Paletas K (2009) Non alcoholic fatty liver disease two-hit process: multifactorial character of the second hit. Hippokratia 13, 128.

39. Jia H, Takahashi S, Saito K, et al. (2013) DNA microarray analysis identified molecular pathways mediating the effects of supplementation of branched-chain amino acids on CCl4-induced cirrhosis in rats. Mol Nutr Food Res 57, 291-306. 